\title{
3 C s - cleft lip, caries and congenitally missing teeth. Interdisciplinary management.-
}

\author{
Dr.Reshma.Dodawad, Dr.Pavithra.S, Dr.Nazima, Dr.Girish.M.S
}

\begin{abstract}
: clefts of the lip and palate are one of the most common congenital craniofacial abnormalities seen in the practice of pediatric dentistry. It has been observed that these children have a higher caries experience compared to their non - cleft counterparts resulting in early pulpal involvement and gross destruction of teeth. They also have congenital anomalies like missing or supernumerary teeth and abnormal development and mineralization pattern of the tooth germs. The following case report documents the restoration of severely mutilated deciduous teeth and an attempt to save the second deciduous molars with missing permanent successors.
\end{abstract}

Key words: caries, cleft lip, missing premolars.

\section{Introduction}

cleft deformities of the lip and palate in children are usually associated with developmental anomalies like hypodontia, hyperdontia, morphological abnormalities and chronological disturbances of the dentition with varying degrees of severity. ${ }^{[2,3]}$ The deciduous and permanent dentition are affected with a greater incidence in the latter. ${ }^{[2]}$ Also, the maxillary arch is more affected than the mandibular with the severity of the anomalies increasing strongly with the severity of the cleft. ${ }^{[6]}$

Due to impaired activity of the surrounding musculature, these patients are susceptible to dentofacial discrepancies. Altered feeding habits and improper oral hygiene predispose them to caries in their early childhood ${ }^{[1]}$ Here we are presenting a case of a full mouth rehabilitation of a nine year old child with repaired unilateral cleft lip, having multiple carious deciduous teeth and several missing permanent teeth.

\section{Case report}

A nine year old male child reported with the chief complaint of pain in the left lower back tooth region. The pain was sharp, spontaneous, localized to the region of the deciduous molars, increased on mastication and occasionally kept the patient awake at night.

The patient s medical history was unremarkable and non - contributory. Extra - oral examination and history taking revealed that the patient had a microform of cleft on the left upper lip which was corrected at 6 months of age. Facial profile of the patient was straight with no gross asymmetry of the face.

On intra - oral examination,it was seen that there was a retained 51 and erupting 11 and 21. Multiple arrested carious lesions were seen i.r.t. 52,53,54,62,63 and 65. Deep carious lesions were seen i.r.t. 64,74,75,84 and 85. The molar relationship was mesial step on both right and left sides with no major discrepancies requiring orthodontic intervention. A panoramic radiograph revealed congenital absence of 31 and missing second premolar tooth buds in all the quadrants. Findings revealed pulpal involvement i.r.t. 64,74,75,84 and 85 .

Treatment was planned accordingly in sequential visits. It was decided to extract the retained deciduous right upper central incisor and 64 due to root resorption. Pulpectomy was done i.r.t. 74 and 84 and obturated with zinc oxide eugenol cement. Since the permanent successors were missing i.r.t. 75 and 85 , these teeth were obturated with gutta percha. Glass ionomer post endodontic restoration followed by stainless steel crowns were given as the permanent restoration for 74,75,84 and 85. Caries was excavated i.r.t. 54 and 55, restored with resin modified glass ionomer cement and stainless steel crowns were given as the final restoration. After removal of caries i.r.t 65 and restoration with resin modified glass ionomer cement, a crown and loop space maintainer was given to preserve the space of 64. Composite build up was done i.r.t. 52,53 and 63 to improve the esthetic appearance of the patient.

Home care instructions and diet counseling was given to the parents and the patient. Recall check-ups were performed every 3 months.

\section{Discussion}

Non - syndromic clefts of the lip and palate are one of the most common malformations seen in the cranio-facial region. ${ }^{[1]}$ Although the frequency varies in different population groups, in some parts of the world, it is more common than even Down's Syndrome. ${ }^{[1]}$ There is an incidence of $24 \%$ of congenitally missing premolars in cleft lip and palate patients as reported by Olin. ${ }^{[2,7]}$ 
The causes of missing teeth can be broadly classified into general and local. General includes a genetic cause, those related to underlying conditions, particularly syndromes like Pierre Robin, Van Der Woude Syndrome with cleft and ectodermal dysplasia. ${ }^{[5,6]}$ Researchers have pointed that a mutation in the homeobox gene MSX1 and PAX 9gene that may be critical to the normal development of specific human teeth. Overall research points to a polygenic mode of inheritance caused by an interaction between the environment and genes. ${ }^{[5,6,10]}$ It has been postulated that specific surgical procedures such as early periosteoplasty or neonatal closure of the hard palate might influence tooth formation. Secondly, the high rate of agenesis near the cleft may be due to a deficiency in blood supply either congenital or secondary to surgery or a lack of stimulation in the underlying mesenchyme. . $^{3,8]}$

Also, the absence of the lateral incisor mesially and /or distally of the cleft could be the result of tissue insufficiency in the medial nasal and /or maxillary process during embryological development. ${ }^{[3]}$ A high prevalence of agenesis outside the cleft area points to a common genetic background for hypodontia and clefts.

Management of hypodontia requires an interdisciplinary approach. In the above case,there was a need to retain the primary molars to act as a natural space maintainer since the patient was not willing to undergo extensive orthodontic or prosthodontic therapy at this stage ${ }^{[4]}$ Primary teeth have one distinct feature in that , they undergo physiological resorption leading to shedding. In the absence of the permanent successors, the resorptive force is missing resulting in a retained tooth. Generally, resorbable materials like ZoE paste, Calcium Hydroxide and Iodoform paste(Vitapex) are used for the obturation of primary teeth. In the event of missing successor teeth, non - resorbable materials like Gutta percha, MTA and Calcium Enriched Mixture (CEM) may be used. ${ }^{[4]}$

Obturation with gutta percha is a more feasible option due to easy availability, good biocompatibility, ease of placement in the canals and cost - effectiveness. MTA/CEM mixture is the material of choice in specific cases of open apices, root resorption and presence of a periapical lesion. It has been seen that root canal space devoid of filling materials become ankylosed and submerged leading to occlusal discrepancies and abnormal force distribution. Space management in hypodontia cases can be managed by closure of edentulous spaces, appropriate redistribution of space or by space maintenance. ${ }^{[4]}$

\section{Conclusion}

Because of the higher prevalence of dental anomalies in individuals with clefts of the lip and palate, early recognition of such abnormalities is crucial for a successful treatment outcome. The opinions and treatment options offered by specialists in an interdisciplinary team goes a long way in enhancing the dental, psychological and general well being of the patient.

\section{References}

[1]. Sheeba Sani, Deepak Sharma. Esthetic and functional oral makeover of a 3 year old cleft palate patient suffering from early childhood caries using unconventional techniques. J Clin Exp Dent 2011;3(2):158-61.

[2]. Yehoshua Shapira DMDa, Erwin Lubit DDSb, Mladen M. Kuftinec D Stom, DMD, ScDc. Congenitally missing second premolars in cleft lip and cleft palate children., Am J Orthod Dentofacial Orthop1999;15(4):396-400.

[3]. M.Okan Akcam, Sehrazat Evirgen, Ozge Uslu and Ufuk Toygar Memikoğlu. Dental anomalies in individuals with cleft lip and/or palate. European Journal of Orthodontics 2010;32:207-213.

[4]. Nagesh Bolla, Balaram D Naik, Sarath Raj Kavuri, Lakshmi Deepa Velagala.Obturation of a Retained Primary mandibular Second molar with Missing Successor Using Gutta-percha:A Case Report. JIDA;2011:5( 2):194-195.

[5]. J H Nunn, N E Carter, T J Gillgrass, R S Hobson, N J Jepson, J G Meechan \& F S Nohl.

[6]. The interdisciplinary management of hypodontia: background and role of paediatric dentistry. British Dental Journal 2003;194(5):245 $-251$.

[7]. Ranta R . A review of tooth formation in children with cleft lip/palat.Am J Orthod Dentofacial Orthop 1986; 90(1):11-8.

[8]. Larson M, Hellquist R, Jakobsson OP. Dental abnormalities and ectopic eruption in patients with isolated cleft palate.Scand J Plast Reconstr Surg Hand Surg 1998; 32(2):203-12.

[9]. Vincent G.Kokich and Vincent G.Kokich.Congenitally missing mandibular second premolars:clinical options. Am J Orthod Dentofacial Orthop2006;130:437-44 .

[10]. Reijo Ranta. Comparison of tooth formation in noncleft and cleft - affected children with and without hypodontia. Journal of Dentistry for children 1982:197-199.

Bartzela TN,Carels CEL,Bronkhorst EM,Ronning E, Rizell S, Kuijpers-Jagtman AM. Tooth agenesis patterns in bilateral cleft lip and palate.Eur J Oral Scin 2010;118:47-52. 


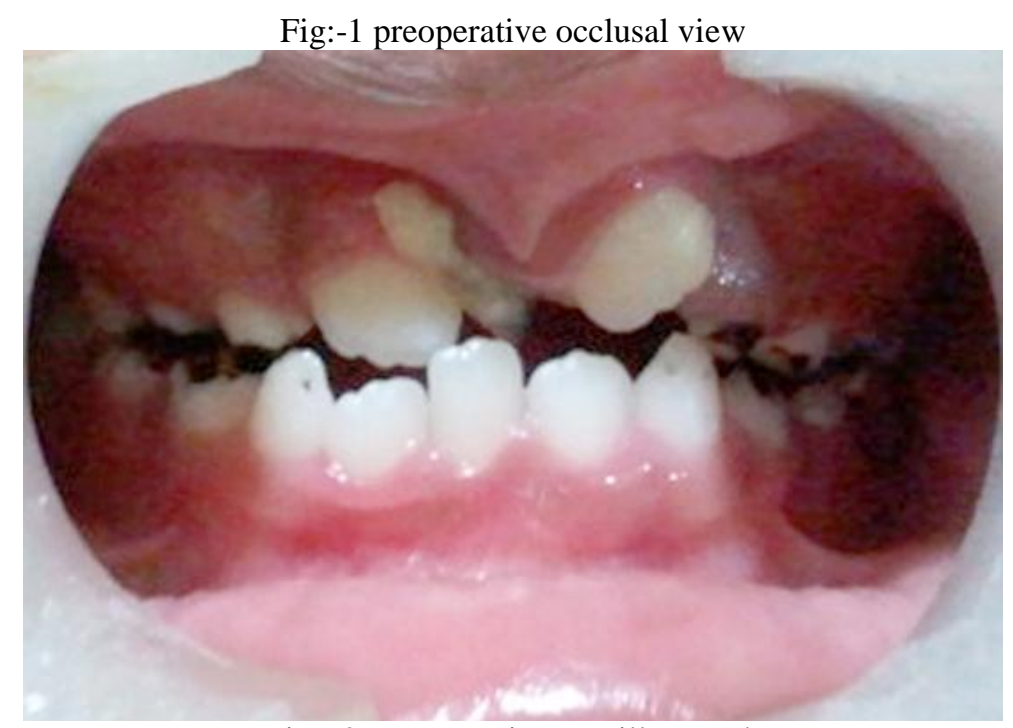

Fig :-2 preoperative maxillary arch

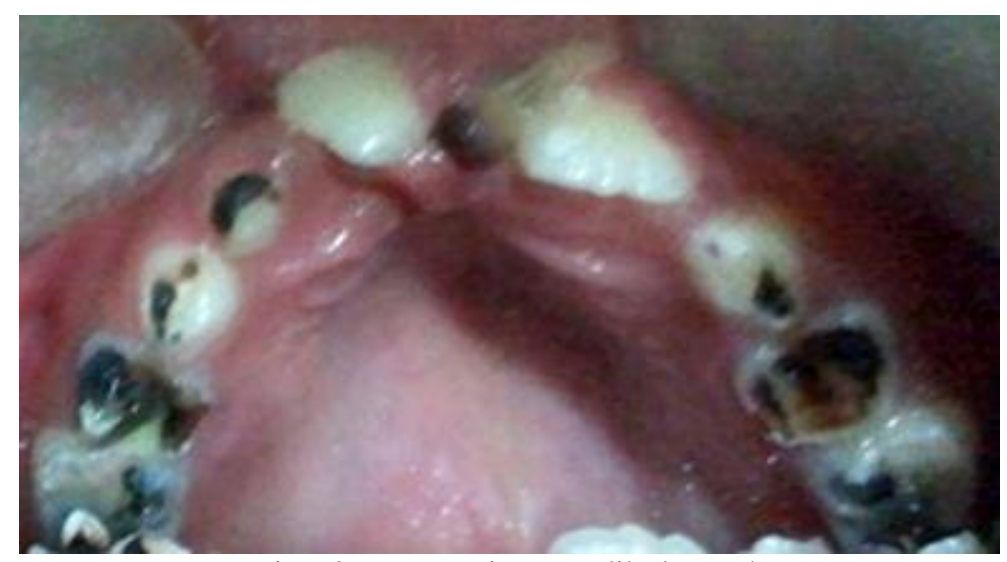

Fig :-3 preoperative mandibular arch

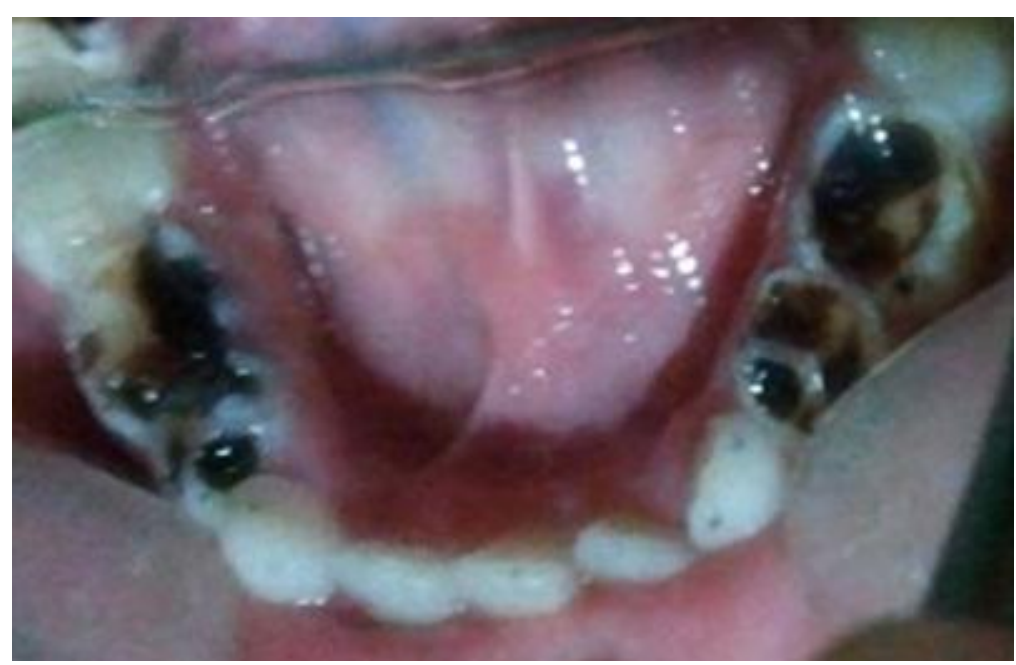

Fig :-4 preoperative OPG 


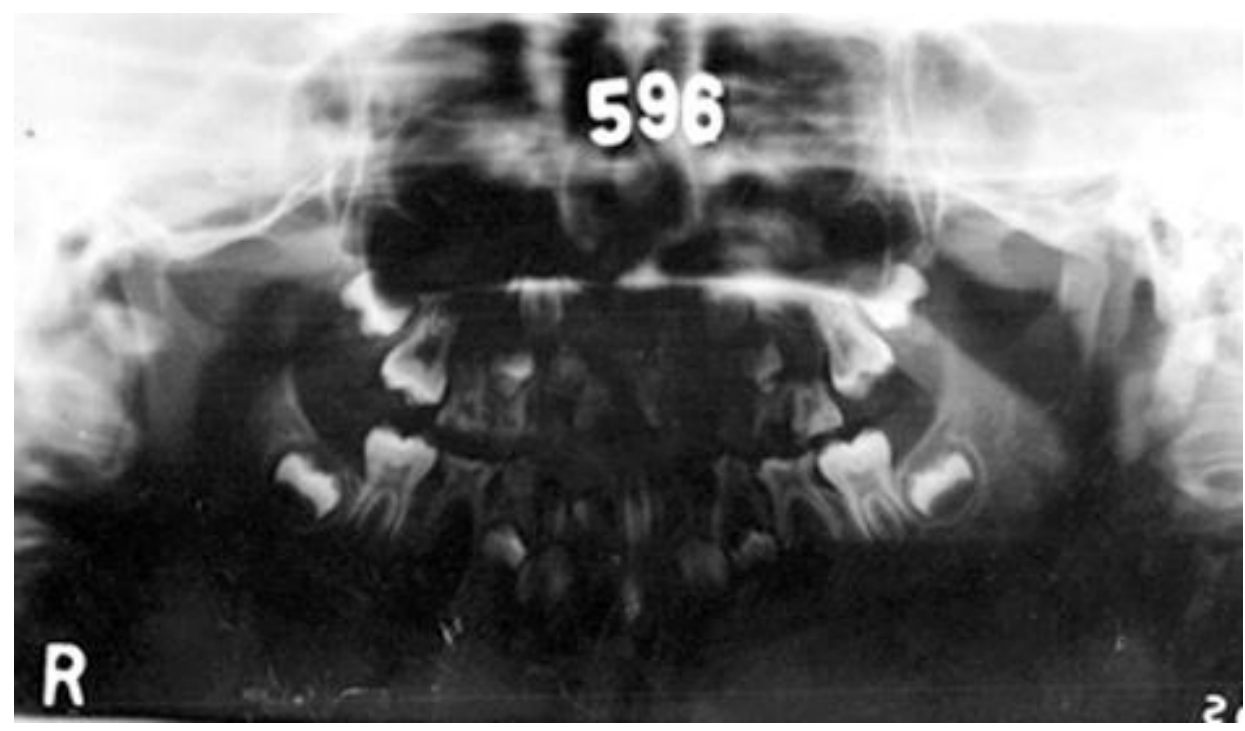

Fig :-5 post operative occlusal view

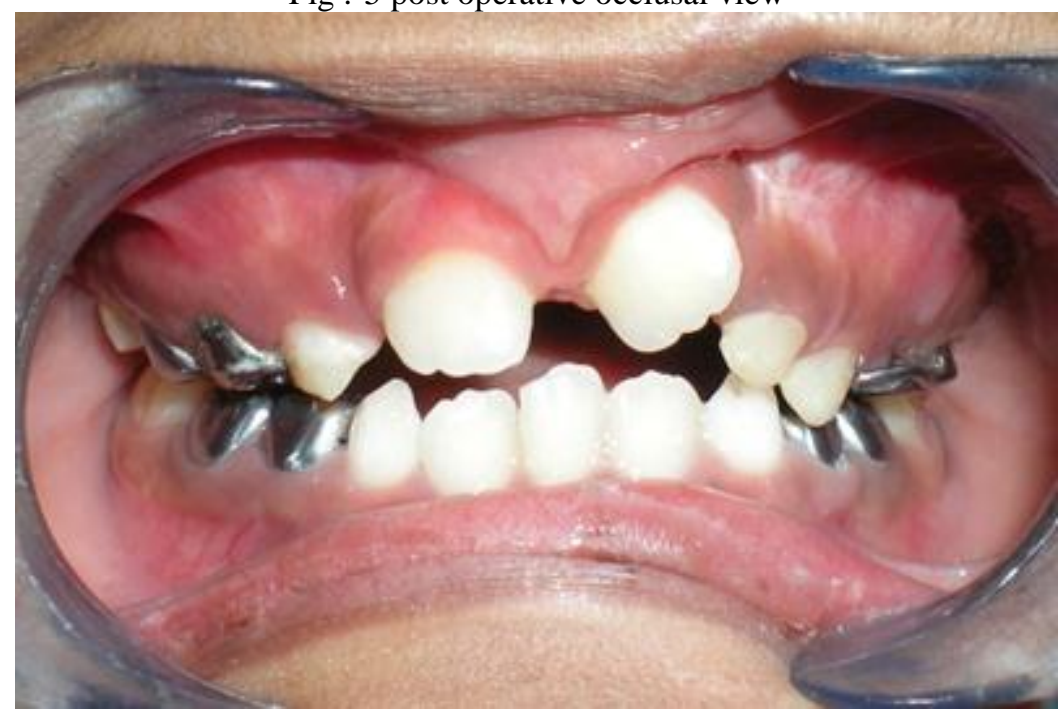

Fig:-6 post operative maxillary arch

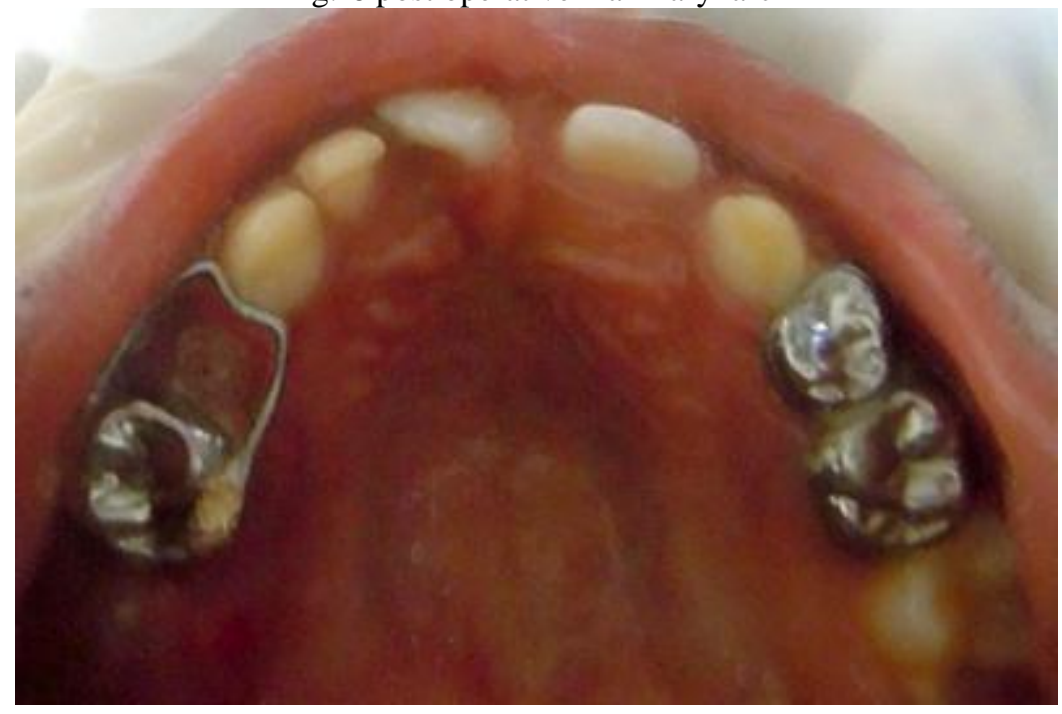


Fig:-7 post operative mandibular arch

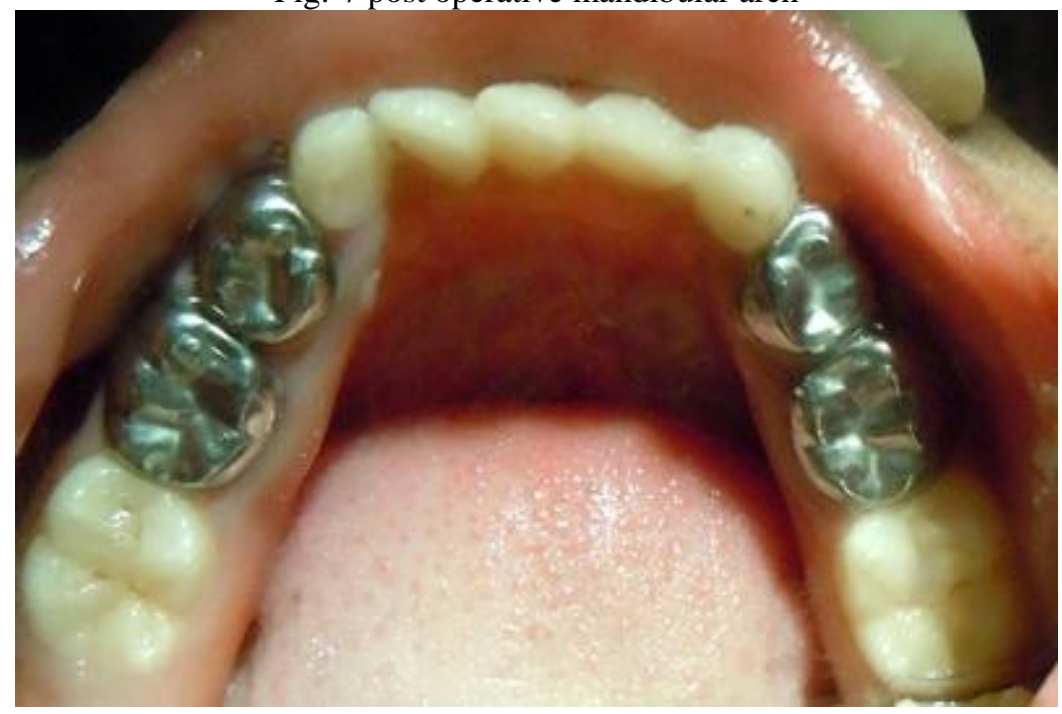

Fig :-8 post operative radiograph

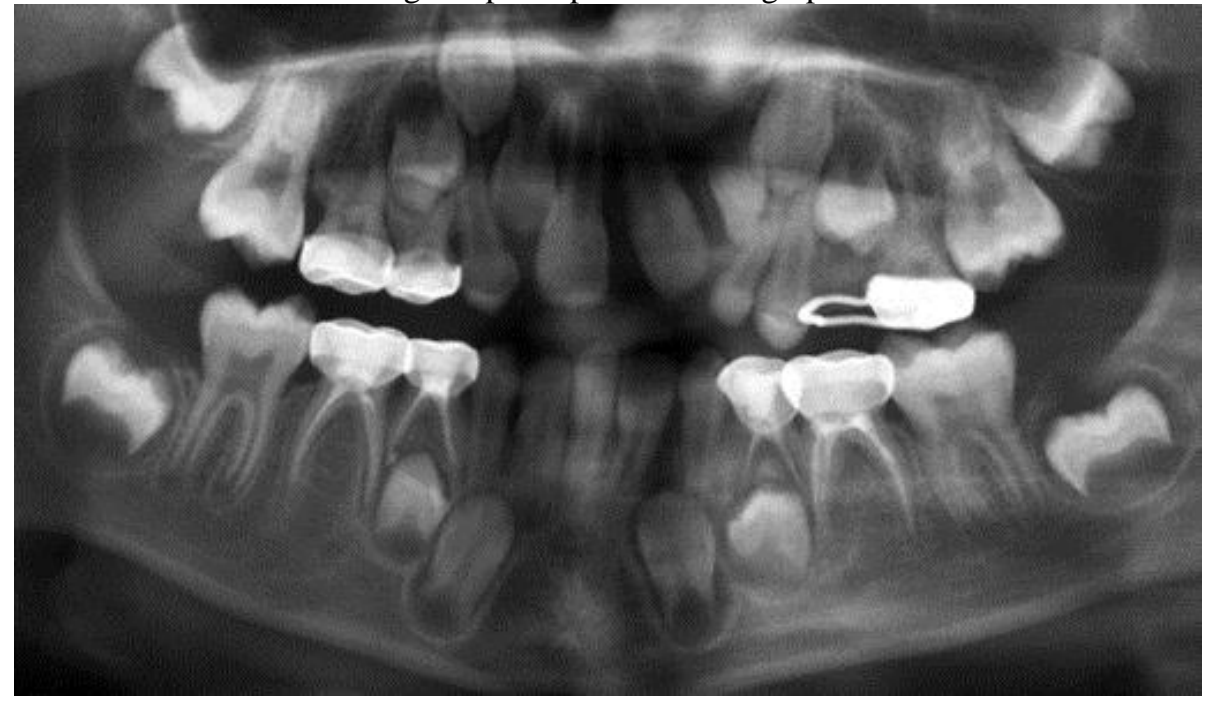

